\title{
Neuronal Oscillations in the Basal Ganglia and Movement Disorders: Evidence from Whole Animal and Human Recordings
}

\author{
William D. Hutchison, ${ }^{1,2}$ Jonathan O. Dostrovsky, ${ }^{2}$ Judith R. Walters, ${ }^{3}$ Richard Courtemanche, ${ }^{4}$ Thomas Boraud, ${ }^{5}$ \\ Joshua Goldberg, ${ }^{6,7}$ and Peter Brown ${ }^{8}$ \\ ${ }^{1}$ Division of Neurosurgery, Toronto Western Hospital, Toronto, Ontario, Canada, M5T 2S8, ${ }^{2}$ Department of Physiology, University of Toronto, Toronto, \\ Ontario, Canada, M5S 1A1, ${ }^{3}$ National Institutes of Health, Neurophysiological Pharmacology Section, Bethesda, Maryland 20892, ${ }^{4}$ Department of Exercise \\ Science, Concordia University, Montreal, Quebec, Canada, H3G 1M8, ${ }^{5}$ Centre National de la Recherche Scientifique, Basal Ganglia Unité Mixte de \\ Recherche 5543, 75794 Paris Cedex 16, France, ${ }^{6}$ Department of Physiology, University of Texas at San Antonio, San Antonio, Texas 78249, ${ }^{7}$ The Hebrew \\ University, Hadassah Medical School, Department of Physiology, Jerusalem 91120, Israel, and ${ }^{8}$ Sobell Department of Neurophysiology, Institute of \\ Neurology, London WC1N 3BG, United Kingdom
}

Key words: beta; gamma; globus pallidus; pallidum; oscillator; Parkinson; subcortical

Neuronal oscillations underlie a number of physiological processes, such as respiration, diurnal rhythms of the sleep-wake cycle, and gait. Oscillatory activity can be observed in many different brain regions and can be synchronized across these different regions or nuclei. Oscillatory activity has long been recognized in the electroencephalogram (EEG), in which synchrony between thalamus and cortex can be observed at different frequencies. These oscillations are generally subdivided into types on the basis of their characteristic frequency and location, such as theta $(2-7 \mathrm{~Hz})$, alpha $(7-13 \mathrm{~Hz}$ in visual cortex $)$, beta $(11-30 \mathrm{~Hz})$, gamma $(30-80 \mathrm{~Hz})$, and $\mathrm{mu}(7-12 \mathrm{~Hz}$, sensorimotor). Recent studies in animals and humans have revealed the existence of several types of oscillatory activity in the various nuclei of the basal ganglia and, although still poorly understood, are believed to play an important function in both the normal physiology and pathophysiology of this system. This mini-symposium will describe the findings of recent studies that have examined various aspects of oscillatory activity in the basal ganglia.

In the past decade, there has been an increase in basal ganglia surgery for movement disorders, primarily for Parkinson's disease, but also dystonia and Huntington's disease, which has provided a unique opportunity for neurophysiologists such as William Hutchison and Jonathan Dostrovsky to probe these subcortical structures in the clinical setting. Although previous surgeries involved the stereotactic placement of lesions in the brain, the era of neuroablative procedures has given way to "neuroaugmentive procedures" involving chronic indwelling electrodes implanted for deep brain stimulation. Many centers use microelectrodes to map the basal ganglia targets in the internal globus pallidum (GPi) and, more recently, the subthalamic nucleus (STN). During the course of these mapping procedures, it

Received Aug. 16, 2004; revised Sept. 1, 2004; accepted Sept. 6, 2004

Correspondence should be addressed to William D. Hutchison, Division of Neurosurgery, Toronto Western Hospital, 399 Bathurst Street MP11-308, Toronto, Ontario, Canada, M5T 2S8. E-mail: whutch@uhnres.utoronto.ca. DOI:10.1523/JNEUROSCI.3366-04.2004

Copyright $\odot 2004$ Society for Neuroscience $\quad$ 0270-6474/04/249240-04\$15.00/0 became evident that oscillatory activity could be detected in the firing of individual neurons in these structures, particularly in the patients with tremor (Hutchison et al., 1997, 1998; Levy et al., 2000, 2002a,b), and this was also reflected in the recordings of rhythmic activity in local field potentials from the relatively large contacts of the deep brain stimulation leads (Levy et al., 2002). Before and concurrent with this clinical work, animal models of Parkinson's disease, such as the 6-hydroxydopamine (6-OHDA) rat model and the 1-methyl-4-phenyl-1,2,3,6-tetrahydropyridine (MPTP) monkey model, have made, and continue to make, a substantial contribution to our knowledge of the underlying neurophysiological changes that give rise to the pathophysiology of the disease (Raz et al., 1996, 2000; Ruskin et al., 1999b, 2003; Goldberg et al., 2004). These changes include increases in firing rate, a tendency to fire in a more irregular pattern, and abnormal oscillatory synchronization.

The canonical model of basal ganglia dysfunction proposes that alterations in neuronal firing rates underlie the spectrum of movement disorders. For the specific example of Parkinson's disease, there is decreased activity of the inhibitory direct pathway connecting the basal ganglia striatal input to its output in the GPi and increased activity in the so-called indirect pathway via the external globus pallidum (GPe) and STN (Fig. 1). The net effect of this imbalance between the pathways is to elevate the firing rate of inhibitory neurons in GPi that project to important premotor structures, such as the thalamus. Inhibition of premotor centers explains the symptoms of akinesia and bradykinesia but does not as easily explain tremor or rigidity. However, animal and patient studies report only small increases in GPi firing rates in the order of $10-22 \%$ in the parkinsonian state (Hutchison et al., 1994; Wichmann et al., 1994, 1999; Levy et al., 2001). Another prediction of the rate model is that hyperkinetic movements, such as dystonia and chorea, are related to low firing rates in GPi output neurons, but recent observations indicate that the GPi activity is similar to that in Parkinson's disease (Hutchison et al., 2003). Other studies also question the predictions by this model of 


\section{Parkinson's disease - Rate Model}

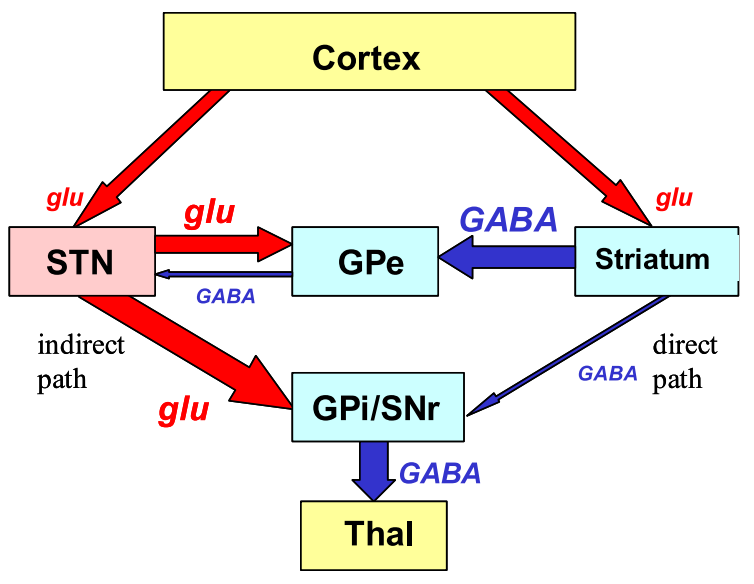

B

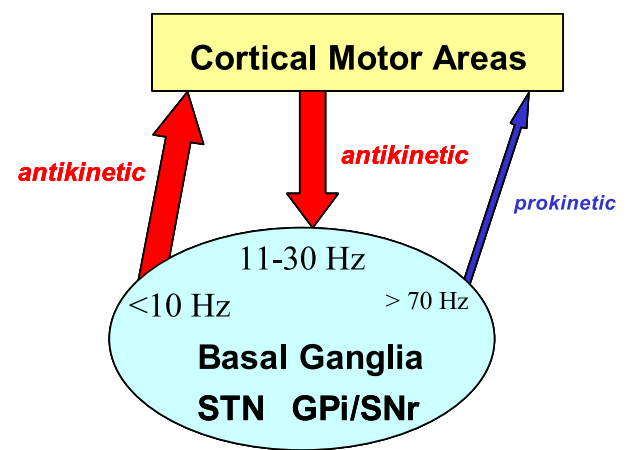

Figure 1. A, Schematic figure of the rate model of Parkinson's disease. The major nuclei of the basal ganglia, the predominant connections, and the neurotransmitters involved are depicted. Information flow is implied by the arrows. In this model, changes in neuronal activity attributable to loss of striatal dopamine are indicated by thick (increased) and thin (decreased) arrows. In Parkinson's disease, activity in the direct pathway from striatum to GPi/SNr is decreased and that in the indirect pathway via GPe and STN is increased, leading to increased firing rates in the output. This inhibits premotor centers, such as thalamus, that facilitate corticospinal motor output. $B$, Schematic figure of the oscillation model of Parkinson's disease. In the absence of dopamine in the striatum, pathological oscillations arise or are enhanced in the basal ganglia, which rhythmically drive other regions or nuclei (depicted by arrows). Frequencies under $10 \mathrm{~Hz}$ (i.e., rest tremor frequency at 3-7 Hz) arise in the basal ganglia and spread to the cortex to produce an antikinetic effect. The STN is driven by oscillations in the cortex in the beta band $(11-30 \mathrm{~Hz})$ that can also be considered antikinetic. Oscillations in the gamma band $(>70 \mathrm{~Hz})$ that facilitate movement (prokinetic) are suppressed or absent in Parkinson's disease (thin blue arrow). Thal, Motor thalamus; glu, glutamatergic (excitatory) pathway; GABA, inhibitory pathway.

changes in firing rates because many basal ganglia neurons have been shown to have intrinsic pacemaker-like properties that sets the rate of firing in the absence of synaptic connectivity (for review, see Bevan et al., 2002).

Effort has now shifted to examining firing patterns and especially synchronous oscillations in the basal ganglia of movement disorder patients to further our understanding of the pathophysiology of abnormal movements (Fig. $1 \mathrm{~B}$ ). One puzzle in the surgical treatment of movement disorders has been why a lesion and electrical stimulation should produce the same therapeutic effect when one obliterates the tissue and the other excites the tissue. The rate model does not reconcile this contradiction, but a model based on network oscillations predicts that either a lesion or deep brain stimulation would disrupt these pathological oscillations, leading to an improvement in symptoms. Pathological oscillatory activity in the alpha frequency $(3-7 \mathrm{~Hz})$ develops in the basal ganglia network, especially in the GPi and in the STN, after MPTP treatment in some monkeys and is also present in the GPi and STN of Parkinson's disease patients with tremor at rest. In addition to tremor-related oscillations, higher-frequency oscillations in the beta range $(15-25 \mathrm{~Hz})$ can be observed in the STN and pallidum even in patients without tremor. However, the relationship between pathological oscillatory activity and the Parkinson's disease symptoms such as bradykinesia and akinesia is unclear.

In addition to the alpha and beta range oscillations, slower oscillations in basal ganglia activity have been recorded by Judith Walters in the 6-OHDA rat model. Their relationship to normal motor control or Parkinson's disease pathophysiology is less readily understood. Connectivity modeling based on the STNGPe network indicates that slow oscillations may also arise as a property of neuronal networks (Terman et al., 2002). Two types of slow oscillation have been studied. Slow oscillations at $0.3-2$ $\mathrm{Hz}$ are often observed in basal ganglia recordings of both local field potential and single-unit activity in anesthetized rat preparations. Ultraslow, multisecond oscillations $(2-60 \mathrm{sec}$ and longer) are frequently seen in basal ganglia recordings from awake immobilized and partially restrained rats. Alterations in dopamine receptor stimulation induce dramatic changes in the properties of these oscillations and the relationships between single-unit activity and local field potentials ( $\mathrm{Hu}$ et al., 2003, 2004). The slow oscillations appear to be more prominent in the firing pattern of basal ganglia neurons of dopamine-depleted rats and are correlated with the local field potentials recorded in cortex and basal ganglia. In contrast, the ultraslow oscillations appear to be enhanced by increases in dopaminergic stimulation, with a greater degree of correlation both within and between various basal ganglia nuclei [GP and substantia nigra pars reticulata $(\mathrm{SNr})]$ on these multisecond timescales (Ruskin et al., 1999a,b, 2003). Lesioning the STN was not found to alter the genesis or transmission of these ultraslow oscillations but did affect dopamine agonist-induced modulation of mean firing rate, oscillatory period, and phase relationship between GP and SNr. These data further support a role for dopamine in modulating coherent oscillatory activity in the basal ganglia and for the STN in shaping the effects of dopamine receptor stimulation on basal ganglia output.

The general view from the movement disorders literature is that basal ganglia oscillations are pathological, but Richard Courtemanche has studied a possible normal physiological function for such oscillations in basal ganglia networks in relation to cortical control of movement (Courtemanche et al., 2003). In the study to be discussed, simultaneous recordings were made with up to eight microelectrodes placed in different parts of the striatum of normal monkeys at rest. Oscillatory activity of the local field potentials in the beta band was observed and was synchronized across many or all of these electrodes, indicating a global synchronization in the striatum. This oscillatory activity was different from simultaneously recorded cortical frontal eye field local field potentials. Single-unit recordings from these same electrodes showed that some cells tended to fire at a specific phase value of the local field potential. Monkeys were also trained to make saccadic eye movements to visual targets, and, as the monkeys performed saccades, the beta-band oscillations decreased across all electrodes. Local field potential synchronization across sites also showed a task-related decrease, yet only for sites that were neuronally engaged in the task, as evidenced by local mul- 
tiunit saccade-related activity. This pattern suggests that, as small focal zones become activated, they become disengaged from general local field potential synchrony during the eye movements. A role for these oscillations during normal motor behavior is suggested by these findings, but it is notable that, during the oculomotor fixation before saccades were made, high levels of synchrony were maintained. Comparisons of spike timing and the simultaneously recorded local field potential oscillations, made as the monkeys rested, showed that some striatal neurons fired in-phase with the oscillations. In pathological conditions, such as the case of dopamine depletion, such oscillations probably entrain more neurons to give rise to pathological oscillatory spike activity. If so, the local field potential oscillations could serve the function of filtering striatal input-output transmission.

Although pathological basal ganglia activity has been well characterized in studies of parkinsonian patients or animal models of the disease, the dynamics of the modifications undergone by basal ganglia neuronal activity during the evolution period of the disease are not known yet. The work presented by Thomas Boraud characterized the evolution of the spontaneous and movement related activity in the GPi during a slow dopamine depletion induced by daily injections of small doses of MPTP. Similar to the findings of Raz et al. (2000), the average firing rate of GPi neurons did not vary significantly after MPTP treatment. Oscillatory activity was found in several frequency ranges (4-9 and 11-14 Hz), with discrepancies between both monkeys. One monkey displayed a substantial synchronized oscillatory activity at $\sim 14 \mathrm{~Hz}$, whereas the other displayed mainly oscillations in the 4-9 Hz range. The increase of these correlated oscillatory activities was progressive and monotonic as the disease severity increased with daily injections of MPTP but occurred later than the onset of bradykinesia. These two facts taken together [(1) the inter-individual variability and (2) the lack of correlation between the occurrence of motor impairment and the significant increase of synchronized oscillations] argue against a direct relationship between the pathological oscillatory activity and parkinsonian bradykinesia. However, it raises the question of the relationship between these $4-9$ and $11-14 \mathrm{~Hz}$ oscillations and parkinsonian tremor because tremor onset can occur simultaneously or even can precede bradykinesia in parkinsonian patients (Agid, 1991).

A recent study by Joshua Goldberg set out to examine the relationship between neuronal synchronization and local field potentials in the basal ganglia of MPTP primates (Goldberg et al., 2004). Because cortical local field potentials are a reflection of synchronous neuronal activity that gives rise to the waveforms of the EEG, it is likely that the local field potentials recorded in the basal ganglia reflect synchronous spiking activity in these nuclei. Enhanced neuronal synchrony is an established correlate of parkinsonism (Raz et al., 1996, 2000; Hurtado et al., 1999; Levy et al., 2000, 2002; Goldberg et al., 2002). Using the correlation-based method of partial spectra analysis, this study demonstrated that the abnormal oscillatory $(\sim 10 \mathrm{~Hz})$ correlations between pairs of neurons in the basal ganglia in the MPTP condition could be statistically accounted for to a large extent by the coupling of each neuron to the local field potentials. One interpretation of this finding is that, in the parkinsonian condition, the basal ganglia and cortex become more closely entrained by global brain dynamics, which are reflected in the widespread local field potentials.

One hypothesis put forth by Peter Brown is that the oscillations in the beta band are enhanced to such an extent in Parkinson's disease and that voluntary movements are not generated because the motor command for initiation cannot override the enhanced oscillatory state (Fig. $1 B$ ). The desynchronization in beta band required to initiate movement cannot "break through" the elevated threshold, leading to the poverty of movement characteristic of the disorder. In this model, both dopaminergic medication and STN stimulation are hypothesized to decrease the pathological oscillations and facilitate movement in parkinsonian patients by decreasing the beta band and enhancing the gamma band. Several studies from Dr. Brown's group have supported this theory (Brown, 2003). In one recent study, Parkinson's disease patients made voluntary movements on a go/no-go task while local field potential recordings were made from the contacts in STN (Kuhn et al., 2004). Beta band activity decreased just before movement onset on the "go" tasks in which movements were executed, and the onset of the desynchronization correlated with reaction time latencies on the task. In the no-go task, there was only a transient decrease in beta power, which rebounded. These results draw a closer link between the generation of voluntary movement and the beta-band desynchronization in STN.

Following on from this hypothesis, electrical stimulation at 20 $\mathrm{Hz}$ should produce an enhancement of beta synchronization in the basal ganglia, which should exacerbate Parkinson's disease symptoms ("antikinetic"), and stimulation in the $60-80 \mathrm{~Hz}$ range should enhance movements ("prokinetic"). Indeed, recent work from Brown's group indicates that STN stimulation at 20 $\mathrm{Hz}$ increases GPi synchrony (Brown et al., 2004). Whether this increased synchrony is associated with an anti-kinetic effect was not directly determined, because assessment of motor behavior in these patients would have resulted in desynchronization at beta-band frequencies and confounded the measurement of synchrony. Stimulation of the STN at higher frequencies $(>70 \mathrm{~Hz})$ had the opposite effect on GPi, that is, it suppressed the spontaneous ongoing local field potential oscillations in the $11-30 \mathrm{~Hz}$ range. High-frequency STN stimulation is well known to produce relief of Parkinson's disease motor symptoms. Because much of this evidence is correlative and circumstantial, additional studies in patients and experimental models of Parkinson's disease are warranted (Brown et al., 2004).

In summary, it is becoming increasingly clear that the basal ganglia sustain certain cortically derived oscillations, as detected in the recordings from individual neurons and local field potentials. The functional significance of oscillations in this range of frequencies remains to be elucidated and will shed light on the pathophysiology of movement disorders and solve some of the puzzles of therapeutic surgical intervention.

\section{References}

Agid Y (1991) Parkinson's disease: pathophysiology. Lancet 337:1321-1324.

Bevan MD, Magill PJ, Terman D, Bolam JP, Wilson CJ (2002) Move to the rhythm: oscillations in the subthalamic nucleus-external globus pallidus network. Trends Neurosci 25:525-531.

Brown P (2003) Oscillatory nature of human basal ganglia activity: relationship to the pathophysiology of Parkinson's disease. Mov Disord 18:357-363.

Brown P, Mazzone P, Oliviero A, Altibrandi MG, Pilato F, Tonali PA, Di Lazzaro V (2004) Effects of stimulation of the subthalamic area on oscillatory pallidal activity in Parkinson's disease. Exp Neurol 188:480 - 490.

Courtemanche R, Fujii N, Graybiel AM (2003) Synchronous, focally modulated beta-band oscillations characterize local field potential activity in the striatum of awake behaving monkeys. J Neurosci 23:11741-11752.

Goldberg JA, Boraud T, Maraton S, Haber SN, Vaadia E, Bergman H (2002) Enhanced synchrony among primary motor cortex neurons in the 1-methyl-4-phenyl-1,2,3,6-tetrahydropyridine primate model of Parkinson's disease. J Neurosci 22:4639-4653. 
Goldberg JA, Rokni U, Boraud T, Vaadia E, Bergman H (2004) Spike synchronization in the cortex-basal ganglia networks of parkinsonian primates reflects global dynamics of the local field potentials. J Neurosci 24:6003-6010.

Hu D, Itoga CA, Bergstrom DA, Walters JR (2003) Local field potentialspike train relationships in substantia nigra pars reticulata are modulated by dopamine cell lesion in urethane-anesthetized rats. Soc Neurosci Abstr 29:273.3.

Hu D, Itoga CA, Bergstrom DA, Parr-Brownlie LC, Ghiglieri V, Walters JR (2004) Increased patterned activity in basal ganglia output in anesthetized rats after dopamine loss is organized by coordinated oscillations in the indirect pathway nuclei. Soc Neurosi Abstr 30:309.8.

Hurtado JM, Gray CM, Tamas LB, Sigvardt KA (1999) Dynamics of tremorrelated oscillations in the human globus pallidus: a single case study. Proc Natl Acad Sci USA 96:1674-1679.

Hutchison WD, Lozano CA, Davis KD, Saint-Cyr JA, Lang AE, Dostrovsky JO (1994) Differential neuronal activity in segments of globus pallidus in Parkinson's disease patients. NeuroReport 5:1533-1537.

Hutchison WD, Lozano AM, Tasker RR, Lang AE, Dostrovsky JO (1997) Identification and characterisation of neurons with tremor-frequency activity in human globus pallidus. Exp Brain Res 113:557-563.

Hutchison WD, Allan RJ, Opitz H, Levy R, Dostrovsky JO, Lang AE, Lozano AM (1998) Neurophysiological identification of the subthalamic nucleus in surgery for Parkinson's disease. Ann Neurol 44:622-628.

Hutchison WD, Lang AE, Dostrovsky JO, Lozano AM (2003) Pallidal neuronal activity: implications for models of dystonia. Ann Neurol 53:480-488.

Kuhn AA, Williams D, Kupsch A, Limousin P, Hariz M, Schneider GH, Yarrow K, Brown P (2004) Event-related beta desynchronization in human subthalamic nucleus correlates with motor performance. Brain 127:735-746.

Levy R, Hutchison WD, Lozano AM, Dostrovsky JO (2000) High-frequency synchronization of neuronal activity in the subthalamic nucleus of parkinsonian patients with limb tremor. J Neurosci 20:7766-7775.

Levy R, Dostrovsky JO, Lang AE, Sime E, Hutchison WD, Lozano AM (2001) Effects of apomorphine on subthalamic nucleus and globus pallidus in- ternus neurons in patients with Parkinson's disease. J Neurophysiol $86: 249-260$.

Levy R, Ashby P, Hutchison WD, Lang AE, Lozano AM, Dostrovsky JO (2002a) Dependence of subthalamic nucleus oscillations on movement and dopamine in Parkinson's disease. Brain 125:1196-1209.

Levy R, Hutchison WD, Lozano AM, Dostrovsky JO (2002b) Synchronized neuronal discharge in the basal ganglia of parkinsonian patients is limited to oscillatory activity. J Neurosci 22:2855-2861.

Raz A, Feingold A, Zelanskaya V, Vaadia E, Bergman H (1996) Neuronal synchronization of tonically active neurons in the striatum of normal and parkinsonian primates. J Neurophysiol 76:2083-2088.

Raz A, Vaadia E, Bergman H (2000) Firing patterns and correlations of spontaneous discharge of pallidal neurons in the normal and the tremulous 1-methyl-4-phenyl-1,2,3,6-tetrahydropyridine vervet model of parkinsonism. J Neurosci 20:8559-8571.

Ruskin DN, Bergstrom DA, Kaneoke Y, Patel BN, Twery MJ, Walters JR (1999a) Multisecond oscillations in firing rate in the basal ganglia: robust modulation by dopamine receptor activation and anesthesia. J Neurophysiol 81:2046-2055.

Ruskin DN, Bergstrom DA, Walters JR (1999b) Multisecond oscillations in firing rate in the globus pallidus: synergistic modulation by D1 and D2 dopamine receptors. J Pharmacol Exp Ther 290:1493-1501.

Ruskin DN, Bergstrom DA, Tierney PL, Walters JR (2003) Correlated multisecond oscillations in firing rate in the basal ganglia: modulation by dopamine and the subthalamic nucleus. Neuroscience 117:427-438.

Terman D, Rubin JE, Yew AC, Wilson CJ (2002) Activity patterns in a model for the subthalamopallidal network of the basal ganglia. J Neurosci 22:2963-2976.

Wichmann T, Bergman H, DeLong MR (1994) The primate subthalamic nucleus. III. Changes in motor behavior and neuronal activity in the internal pallidum induced by subthalamic inactivation in the MPTP model of parkinsonism. J Neurophysiol 72:521-530.

Wichmann T, Bergman H, Starr P, Subramanian T, Watts RL, DeLong MR (1999) Comparison of MPTP-induced changes in spontaneous neuronal discharge in the internal pallidal segment and in the substantia nigra pars reticulata in primates. Exp Brain Res 125:397-409. 